\title{
Shadowgraphic Imaging of Fibre-Delivered Pulsed IR Laser-Induced Heat Transfer across Thin Aluminized Polymer Film
}

\author{
Mohammad E. Khosroshahi ${ }^{1,2,3}$ \\ ${ }^{1}$ Laser \& Nanobiophotonics Laboratory, Faculty of Biomedical Engineering, Amirkabir University of Technology, Tehran, Iran \\ ${ }^{2}$ MIS-Electronics, Nanobiophotonics \& Biomedical Research Laboratory, Richmond Hill, Canada \\ ${ }^{3}$ Department of Mechanical and Industrial Engineering, University of Toronto, Toronto, Canada \\ Email:khosrom@mie.utoronto.ca
}

How to cite this paper: Khosroshahi, M.E. (2018) Shadowgraphic Imaging of Fibre-Delivered Pulsed IR Laser-Induced Heat Transfer across Thin Aluminized Polymer Film. Optics and Photonics Journal, 8, 75-89.

https://doi.org/10.4236/opj.2018.84008

Received: February 12, 2018

Accepted: April 8, 2018

Published: April 11, 2018

Copyright $\odot 2018$ by author and Scientific Research Publishing Inc. This work is licensed under the Creative Commons Attribution International License (CC BY 4.0).

http://creativecommons.org/licenses/by/4.0/

\section{c) (i) Open Access}

\begin{abstract}
Shadowgraphic imaging was employed to investigate the mid-IR laser induced heat transfer through a double layer thin film. The effect of thin metal coat on the polymer film enhanced the transfer of heat and shock waves due to rapid thermal expansion and the explosive evaporation of the thin fluid layer. Sixty two percent of deposited heat expended for water enthalpy and 38\% for other factors. A power of $8.8 \mathrm{~kW}$ was launched at the surface of aluminium. The thermal coupling of $45 \%$ further reduced the input energy to the film and the non-adiabatic heat diffusion (i.e., $X_{T} \gg \delta_{0 A}$ ) was transmitted instantaneously within the metal with very small loss. The temperature at the surface of the film was determined $\approx 301 \mathrm{~K}$, well below the aluminium melting point. The Biot number showed that the metal as single layer and the whole film as double layer satisfies the thermally thin film (i.e., «0.1). Considering the Newtons's law of cooling, the overall film heat transfer coefficient was found 3 $\mathrm{k} \mathrm{W} \cdot \mathrm{m}^{-2} \cdot \mathrm{K}^{-1}$ equivalent of $3.3 \times 10^{-3} \mathrm{~W} \cdot \mathrm{m}^{2} \cdot \mathrm{K}^{-1}$ thermal resistance. The analysis of images indicated a reducing percentage of heat transfer as a function of delay time based on the comparison of volume ratios. A calculated power of $\approx 3$ $\mathrm{kW}$ was transmitted from the rear side of the film sufficient to thermalize the surrounding water layer and form vapor bubble.
\end{abstract}

\section{Keywords}

Thin Film, Heat Transfer, IR Laser, Optical Fibre, Shadowgraphy

\section{Introduction}

Pulsed laser interaction with materials whether organic or inorganic and hence 
understanding the underlying mechanism in the process is important in various science and engineering applications such as laser material processing where welding, drilling, cutting and surface modifications or cleaning involves the removal material and for non-removal applications including medical diagnosis and industrial non-destructive testing, one requires process monitoring and on-line quality control [1]-[6]. Also, the applications of cavitation bubbles, shock waves and liquid jet impinging on the tissue have generated much interest in biomedical research [7] [8] [9] [10]. Depending on the laser intensity and pulse duration, the heating process can be classified as conduction limited or non-conduction limited heating. In the former case heating substrate material remains in the solid phase while phase change (melting and vaporization) occurs in the latter. For metals and metal-like materials, three mechanisms are generally referred to when considering thermal processes: Normal vaporization, normal boiling and explosive boiling (or phase explosion). For nanosecond laser pulses, the region of normal vaporization gives way to phase explosion with increasing laser fluence when the irradiated matter approaches the thermodynamic critical point [4] [6].

In recent times a great interest has been shown in under water laser processing due to number of interesting features such as better process efficiency, quality and faster processing. For example, enhanced adhesion of metal films on polyethylene terephthalate (PET) using $\mathrm{KrF}$ excimer laser [11], investigation of shock waves generated by $\mathrm{XeCl}$ excimer laser ablation of sheet polyimide confined in water [12], irradiation of solid targets in water with Nd:YAG laser to fabricate $\mathrm{Ag}, \mathrm{Au}, \mathrm{Ni}$ and $\mathrm{Cu}$ nanoparticles [13] and interaction of IR laser with metal targets in water to investigate the process involved in the treatment [14] and drilling thin film sheet [15]. It is known that at low level powers, the laser can be a valuable analytical tool, which allows one to investigate the material properties without damaging the material or changing the properties. One such application is to fabricate thin films as crucial component for various applications such as multilayer microelectronics, optical devices and micro-actuators in micro-electromechanical devices. Lasers have been used to investigate the spallation of thin film [16] and the hydrodynamic instabilities of $\mathrm{Al}$ and $\mathrm{Au}$ thin metal melts [17]. The concept and understanding the mechanism of heat transfer through metallic and non-metallic thin films has a crucial role in number of scientific, engineering and biomedical applications. Heat transfer and fluid flow are of great importance and interest in scientific research and one of the fundamental issues which has been the center of focus is the transient temperature field developed during pulsed laser processing particularly in the case of thin films where the damage possibility is a main concern, though the main objective is determined by the application. Since, thin films can be in the form of a single, double and multilayer, thus the problem can be further complicated by number of layers and that each requires a careful analysis. For example, removal of superficial layer without damaging the underneath substrate or in the case of la- 
ser-induced forward transfer (LIFT) which is direct-write method, involves a focusing laser pulse through a transparent substrate (carrier) onto the rear side of a thin film of the material (donor). Another substrate (receiver) upon which the donor material supposed to be deposited is placed close to the coated carrier (target). Another example is phonon transport in dielectric thin films where the heat conduction is highly important and a critical issue in the design of electronic devices and packages. Thin films are also widely utilized in biomedical engineering such as tissue engineering and bioimplants where the films can be used on different surfaces with respect to their physicochemical characteristics such as wettability, reactivity, conductivity and corrosion properties. There are number of techniques which can be used to study the heat transfer each with its advantages and disadvantages. Among these optical technique is considered as flexible non-intrusive tool for real time visualization of fluid motion and temperature distribution over the whole field. Generally, an optical technique is divided according to their principles: thermal radiation, light scattering from particles, interaction of fluid flow-solid surface, and change of refractive index in the fluid (e.g. Schlieren and speckle). Some investigators have studied: laser-induced shock waves in water using an interferometric method [18], UV laser ablation of thin metal films by fast photography [19], laser polymer ablation by shadowgraphy [20], the heat transfer in fluid by optical methods [21], IR thermography to study the heat transfer [22], LIFT in thin films using shadowgraphy [23], the laser-driven nanopowders using shadowgraphic technique [24] heat transfer in water using schlieren technique [25] and imaging the metal thin film ablation by dynamic TEM [26]. Based on the previous works and the apparently the lack of information in literature on imaging of both sides of a thin film during IR laser heating, the purpose of this research is to describe the results of laser-induced heat transfer through a double layer aluminized coated Polyethylene terephthalate (PET) thin film under water utilizing shadowgraphy technique.

\section{Experimental}

The experimental setup is shown in Figure 1 where the PET (Maylar, Dupont) foil with typically $50 \mu \mathrm{m}$ thick coated with $80 \mathrm{~nm}$ thick aluminium film was placed inside the water container and irradiated by a home-built multiline hydrogen fluoride (HF) laser wavelengths spanning between $(2.65-2.96 \mu \mathrm{m})$ with the dominant output at $2.75 \mu \mathrm{m}$. Multiline output energies of up to $500 \mathrm{~mJ}$ in a $\approx 400 \mathrm{~ns}$ FWHM pulse with a gaussian profile was obtained at a pulse repetition frequency of $0.2 \mathrm{~Hz}$ [27]. The beam was transmitted via a short fluoride glass optical fibre with $500 \mu \mathrm{m}$ core diameter (Infrared Fibre Systems). Shadowgraphic imaging was used to study the fast bubbles formation and heat transfer at the front and rear side of the film. For this purpose, a $\mathrm{N}_{2}$-pumped dye laser using rhodamine $6 \mathrm{G}, 4 \mathrm{~ns}$ pulse duration and operating at $573 \mathrm{~nm}$ was expanded to provide a $13 \mathrm{~mm}$ diameter collimated beam traversing the water container 
transverse to the fibre. A pulsed delay generator (Stanford-DG535 Model) was used to be simultaneously triggered by the pump HF laser and create a delay time between the pump and the probe dye laser, Figure 2 .

\section{Results}

Figure 3 indicates the variation of optical fibre output fluence with input fluence. As it seen, the output fluence increases linearly up to about $9 \mathrm{~J} \cdot \mathrm{cm}^{-2}$ beyond which exhibits a non-linear behaviour. However, in the experiment a lower value of $\approx 5.5 \mathrm{~J} \cdot \mathrm{cm}^{-2}$ equivalent to $10 \mathrm{~mJ}$ of input energy was used due to fibre longer endurance against higher number of laser pulses.

The shadowgraphs observed for the fibre-film in contact mode under water are shown in Figure 4. Bubble growth is initiated at the fibre tip because of

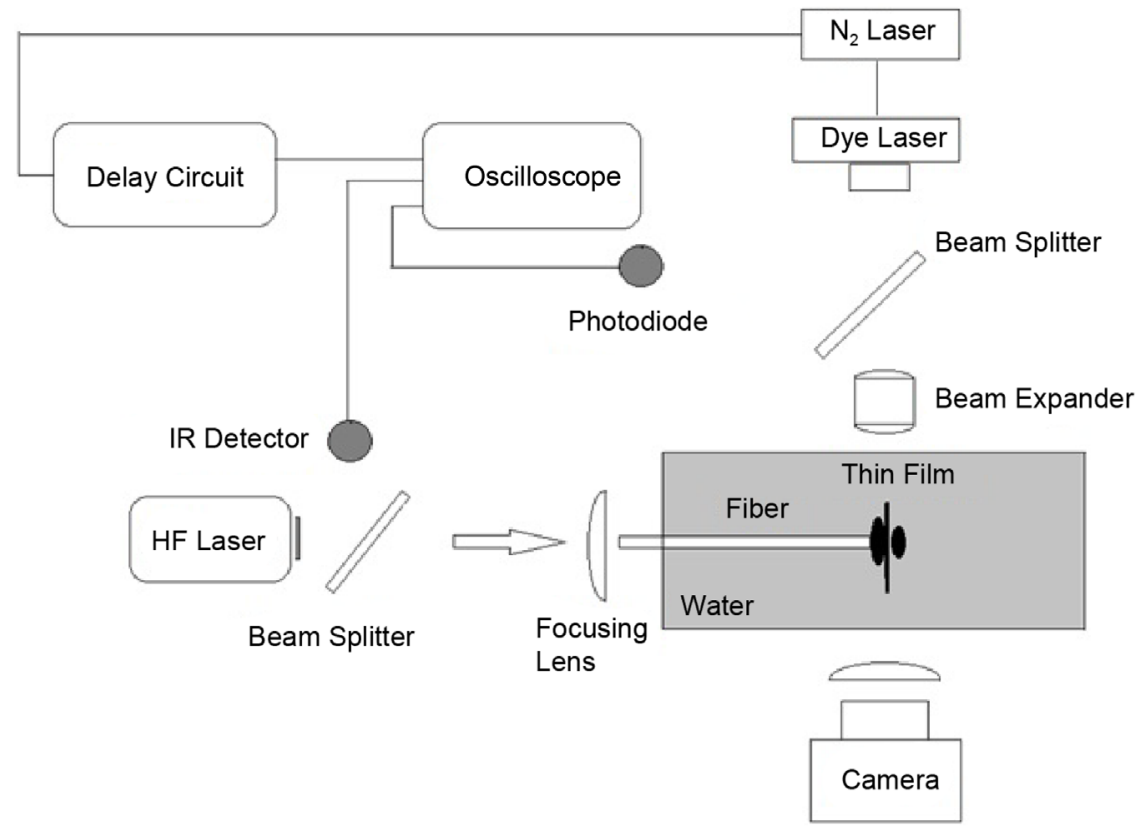

Figure 1. Experimental setup.

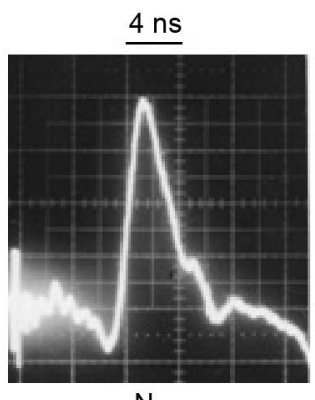

$\mathrm{N}_{2}$

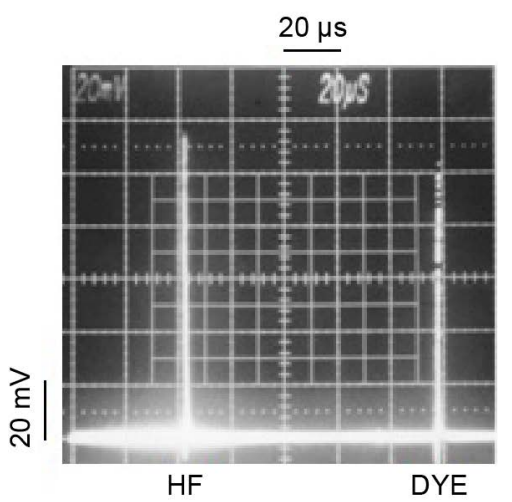

(b)

Figure 2. (a) Pulse duration of $\mathrm{N}_{2}$ laser; (b) An example of HF and Dye lasers pulse synchronization for time-resolved imaging. 


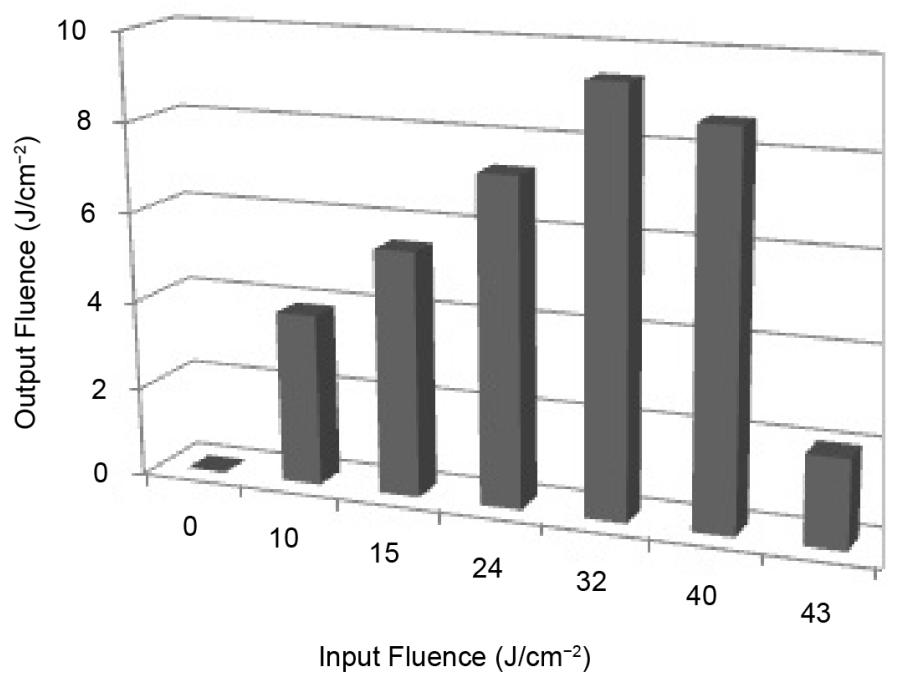

Figure 3. Variation of optical fibre output fluence with input fluence.

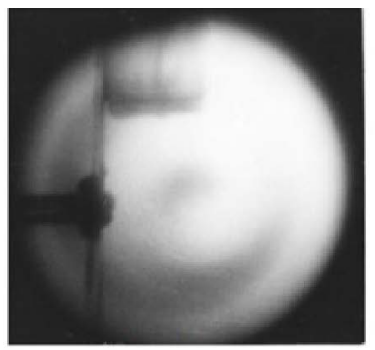

$10 \mu \mathrm{s}$

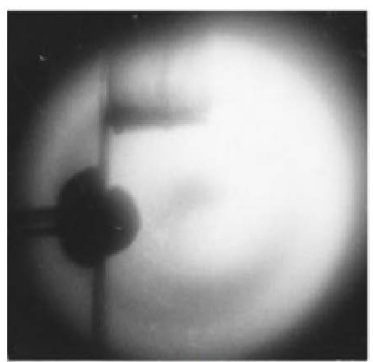

150

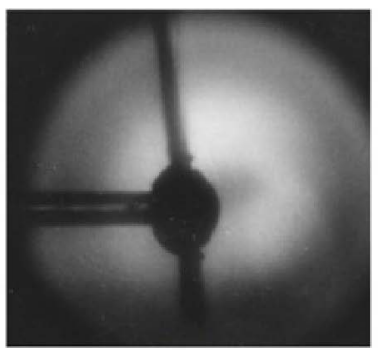

15

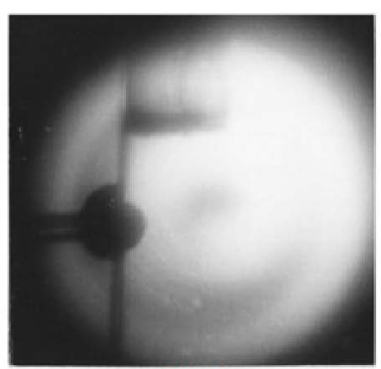

200

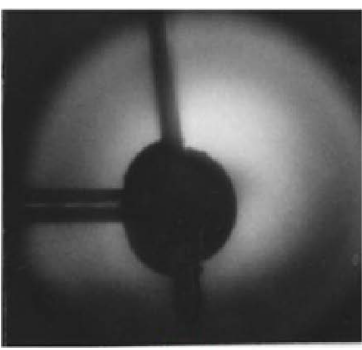

50

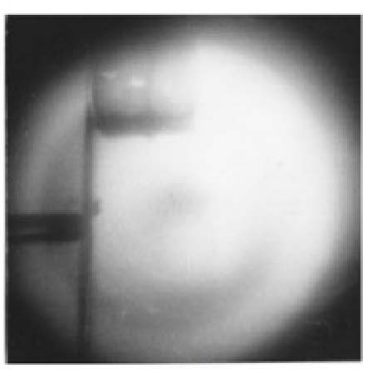

300

Figure 4. Time-resolved shadowgraphic images of laser-induced cavitation bubbles at front and rear side of metal-polymer film. The fibre is in intimate contact with the film.

strong absorption of radiation by water which results in a hot, high-pressure vapour cavity. Evidently, the bubbles initially expand to form approximately a spherical cavity but is somewhat flattened at the fibre tip. A small hemispherical cavity has already developed around the fibre tip at $10 \mu \mathrm{s}$ as a result of high pressure vapor cavity due to strong absorption of the beam, which indicates there exists a very thin layer of water between fibre tip and the metal film because of probably microscopic spatial variation. The hemispherical expansion continuous up to $\sim 250 \mu$ s with a maximum diameter at $50 \mu$ s from there onwards it gradually decreases where it collapses and completely vanishes at $\sim 300$ $\mu$ s. During the expansion and contraction phase, acoustic pressure waves are 
generated within the vapor which then propagate into the surrounding liquid through the interface between vapor and the liquid. Throughout the process the bubble on the rear side of the metal film demonstrated a corresponding behaviour. It was also noticed that at 15 and $50 \mu$ s where the amount of heat transferred is maximum, the film was slightly tilted and pushed away due to the radiation pressure and momentum transfer which then it returns to its original position afterwards.

To study the effect of heat transfer at a distance, the film was moved to about $1 \mathrm{~mm}$ away from the fibre tip, Figure 5 and although the distance was randomly selected, it however indicates that no bubble cavitation is formed at the rear side at this distance indicating the absence of heat transfer. Clearly, for more accurate conclusion in this respect, a detailed measurement and analysis is required.

Figure 6 demonstrates the variation of bubble diameter at both sides of the film as a function of time. The greatest diameter is achieved at $50 \mu \mathrm{s}$ in both cases where it begins to decrease at longer time intervals due to decrease in internal energy of vapor gas molecules against the external pressure to thermodynamically maintain the amount of work done p. $\delta \mathrm{V}$. Therefore, according to first

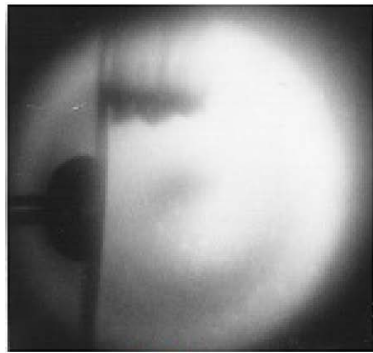

$50 \mu \mathrm{s}$

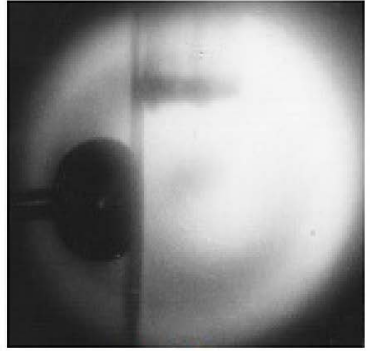

100

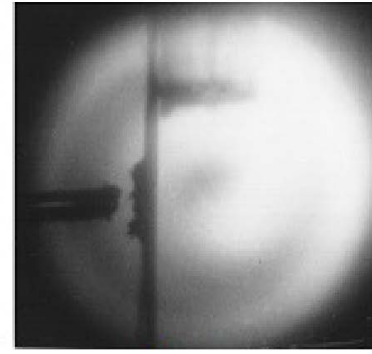

300

Figure 5. Time-resolved shadowgraphic images of laser-induced cavitation bubbles at front and rear side of metal-polymer film at $1 \mathrm{~mm}$ distance. No cavitation bubble is formed at the rear side due to insufficient heat transfer.

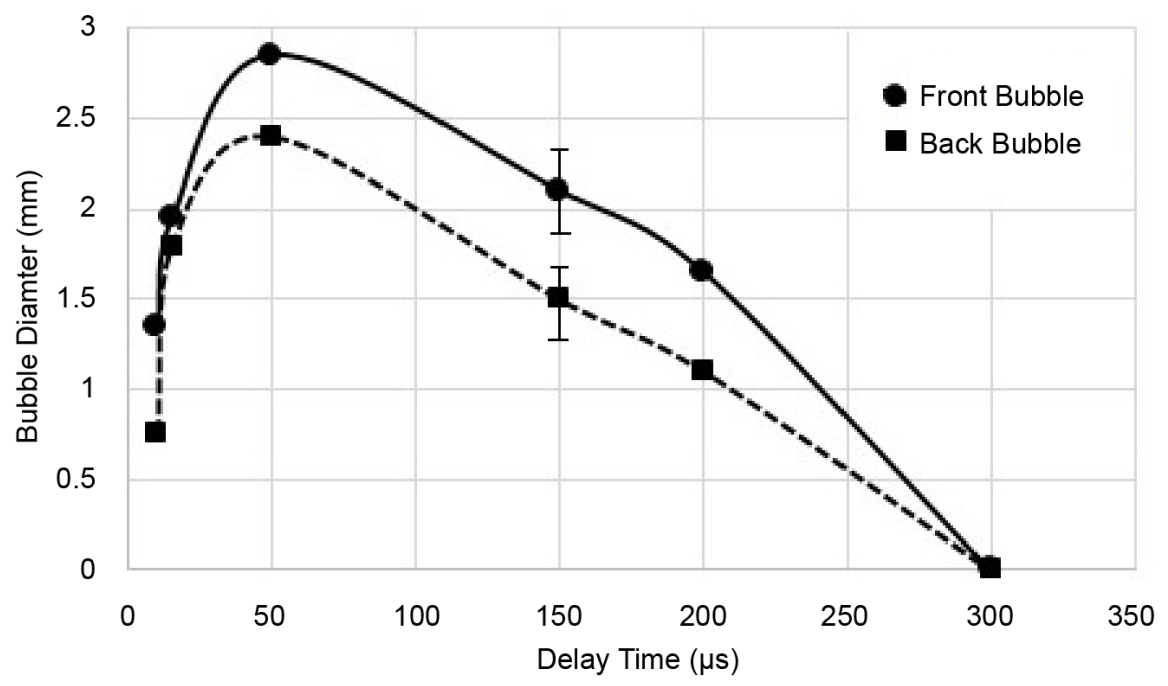

Figure 6. Variation of front and rear bubble diameter with time at $5.5 \mathrm{Jcm}^{-2}$. 
law of thermodynamics for the given amount of heat $\delta \mathrm{Q}(\mathrm{J})$ provided by laser pulse, the internal energy $\delta \mathrm{U}$ is decreased by increasing the bubble volume $\delta \mathrm{V}$ at constant external pressure $\mathrm{p}$.

\section{Discussion and Analysis}

\subsection{Fibre-Water Volume Element}

The interaction of laser beam (Gaussian mode in our case) with liquid and hence its following consequences depends largely on the laser wavelength and type of liquid as they govern the degree of absorption of laser beam, which to first approximation is assumed to behave according to Beer's-Lamberts law within the medium i.e., $\alpha \gg \beta$

$$
I(r, z)=I_{0}(r, z) \cdot e-2 r^{2} / \omega^{2} \mathrm{e}^{-\alpha x}
$$

where $I\left(\mathrm{~W} \cdot \mathrm{m}^{-2}\right)$ is the laser irradiance, $r$ is the radial distance from the center of beam, $\omega(z)$ is the radius at which the field amplitudes fall to $1 / e$ of their axial values, $\alpha\left(\mathrm{m}^{-1}\right)$ is the absorption coefficient and $x(m)$ is the material thickness. At low fluences heating will result in thermoelastic stress and the rapid expanding water produces acoustic effects. At higher fluences boiling occurs i.e., $T \geq 373 \mathrm{~K}$ for $P_{0} \approx 10^{5} \mathrm{~Pa}$. Taking extinction coefficient of water, $k_{e} \approx 0.059$ at $2.75 \mu \mathrm{m}$ [28], it gives $\alpha_{W} \approx 2.7 \times 10^{5} \mathrm{~m}^{-1}$. Thus, the thermal relaxation time is

$$
\tau_{r}=\delta_{0}^{2} / 4 D_{t}
$$

where $\delta_{0} \approx \alpha^{-1} \approx 3.7 \mu \mathrm{m}$ is the optical penetration depth in water and $D_{t} \approx 1.43 \times$ $10^{-7} \mathrm{~m}^{2} \cdot \mathrm{s}^{-1}$ is the water thermal diffusivity, so $\tau_{r} \approx 28 \mu \mathrm{s}$, Also, the thermal diffusion depth is:

$$
X_{T}=\sqrt{4 D_{t} \tau_{p}}
$$

Substituting the above values in Equation (3) yields $X_{T W} \approx 0.45 \mu \mathrm{m}$, where the subscript TW stands for thin water. The simple calculation shows an adiabatic condition where heat is confined at the fibre tip i.e., $\tau_{p} \ll \tau_{r}$ and $\delta_{0} \gg X_{T W}$. Assuming a very thin layer of water $X_{W}$ with a thickness of about $1 \%$ of core diameter i.e., $\mathrm{x}_{\mathrm{w}} \approx 5 \mu \mathrm{m}$ between the fibre and metal film hence giving a thin disc type geometry, so the elemental volume is $\approx 9.8 \times 10^{-4} \mathrm{~mm}^{-3}$. Thus, the output energy of fibre which was measured $\approx 10 \mathrm{~mJ}$ in air is now $E=E_{0} \mathrm{e}^{-\alpha_{w} X_{W}} \approx 2.6$ $\mathrm{mJ}$, corresponding to $3.3 \times 10^{10} \mathrm{~W} \cdot \mathrm{m}^{-2}$. Thus, the amount of heat generated in the volume element of water due to power deposition is $I_{0} \alpha_{w} \approx 9 \times 10^{15} \mathrm{~W} \cdot \mathrm{m}^{-3}$ which corresponds to a power of $P \approx\left(I_{0} \alpha_{w}\right) \delta V \approx 8.8 \mathrm{~kW}$.

Now, if a thin layer of liquid is evaporated in a short timescale by the absorption of laser energy, the increase in pressure on the surface will give rise to shock wave which then will propagate into the thin film. The formation of bubbles proceeds with a front velocity smaller than the shock wave velocity in the medium. Note that the input heating power is divided between number of factors involved in the interaction process mainly to bring the water temperature to boiling point, enthalpy of water $(\Delta H \approx 2252 \mathrm{~kJ} / \mathrm{kg}$ ), superheating, heating the 
film surface part of which is transferred into the film and partly lost by Newton's cooling law. In this case, $\Delta H$ is determined as $\left(I_{0} \alpha_{w} \tau_{p}\right) / \rho_{w} \approx 3600 \mathrm{~kJ} / \mathrm{kg}$ where $\rho_{w}$ $=1000 \mathrm{~kg} \cdot \mathrm{m}^{-3}$ is density of water. So that, $\approx 62 \%$ is expended for boiling and $\Delta H$ and the rest $(\approx 38 \%)$ is utilized for other factors. Also, in our case where $\tau_{p} \gg$ the stress relaxation $\left(\alpha_{w} c_{a}\right)^{-1} \approx 2.5 \mathrm{~ns}$ where $c_{a} \approx 1500 \mathrm{~m} \cdot \mathrm{s}^{-1}$ is acoustic velocity in water then the peak thermoelastic stress is [8]

$$
\sigma_{T}=f \Gamma I_{o} / c_{a}
$$

Substituting the above values and $\Gamma \approx 0.1$ as the Gruneisen constant for water [29] and $f \ll 0.5$ a factor that depends on the precise laser pulse shape in Equation (1) gives $\sigma_{T} \leq 7 \times 10^{4} \mathrm{~Pa}$ i.e., the thermoelastic stress is relatively small due to fact that stress relaxation by the propagation of an acoustic wave across the heated zone occurs on timescale that is much smaller than 400 ns laser pulse duration. Boiling of the heated water layer occurs when the temperature exceeds $\sim 373 \mathrm{~K}$ under conditions where the local pressure in the liquid is $\sim 10^{5} \mathrm{~Pa}$. However, due to the strongly nonequilibrium nature of the interaction it is postulated that substantial superheating takes place i.e., temperature exceeds $T\left(P_{v}\right)=373 \mathrm{~K}$, where $P_{V} \approx 10^{5} \mathrm{~Pa}$ is the vapour pressure that exists under equilibrium conditions. The initial stage of volume growth is limited by the surface tension force and the pressure required to overcome the surface tension is rapidly decreased and explosive vaporization occurs on the surface. The exploding vapor volume growth is restricted by the surrounding water inertia where the vapor gas volume reaches its maximum rate defined by

$$
V(t)=V_{i} t^{3}
$$

where $V_{i}$ is the initial vapor volume at the early stage of growth and $\mathrm{t}$ is time. The maximum velocity when $P_{i} \gg P_{o}$ where $P_{i}$ and $P_{o}$ are the initial and atmospheric pressure respectively, is given by [30]

$$
\rho \dot{R}^{2} \approx \frac{2 P_{i}}{3(1-\gamma)}\left[\left(\frac{R_{i}}{R}\right)^{3 \gamma}-\left(\frac{R_{i}}{R}\right)^{3}\right]
$$

where $\gamma=4 / 3$ and at $\mathrm{d} \dot{R} / \mathrm{d} R=0$ gives maximum when $\dot{R} / R \approx \gamma^{\frac{1}{3(\gamma-1)}}$, then

$$
\rho \dot{R}^{2} \approx \frac{2 P_{i}}{3(1-\gamma)} \times 0.105
$$

using $\rho=1000 \mathrm{~kg} / \mathrm{m}^{-3}$, output fluence of $5.5 \mathrm{~J} \cdot \mathrm{cm}^{-2}$ and $\dot{R} \approx 180 \mathrm{~m} \cdot \mathrm{s}^{-1}$, it gives $P_{i} \approx 1.5 \times 10^{8} \mathrm{~Pa}$.

Despite the initiation of bubble growth, the maximum rate at which energy can be transferred to the vapor is limited by the large difference in volume between the liquid and vapor below the critical point as well as the finite bubble growth velocity. However, if this falls below the rate at which the energy is input from the laser then the liquid temperature will continue to increase and superheating occurs. Therefore, the liquid is heated at approximately constant pressure $\left(10^{5} \mathrm{~Pa}\right)$ to the critical temperature $\left(T_{c}=647 \mathrm{~K}\right)$ at which the distinction 
between liquid and vapor is lost. The pressure then increases and growth of a vapor cavity can take place. The characteristic collapse time of the gas bubble is [30]

$$
\tau_{c}=0.91 R_{m}\left(\rho / p_{0}\right)^{1 / 2}\left(1+P_{b} / P_{0}\right)
$$

where $R_{m} \approx 1.4 \mathrm{~mm}$ is the maximum bubble radius (at $50 \mu \mathrm{s}$ ), $P_{0}$ is the water atmospheric pressure, $P_{b}$ is the pressure in fully expanded bubble and taking $P_{b} / P_{0}$ $\leq 2.7 \times 10^{-2}$ it gives $\tau_{c} \approx 100 \mu \mathrm{s}$. This is clearly, higher than the experimentally observed value i.e. $50 \mu$ s seen in Figure 6, which is accounted on the difference between the experimental and theoretical values. Also, the instability of the cavity during the collapse phase is consistent with the onset of the Rayleigh-Taylor instability for a curved liquid-gas interface [30]. Once, the bubble reached to its maximum radius the velocity of the bubble wall and hence the liquid falls to zero and the potential energy $E_{p}$ of the cavity can be found from the work done on the water [18]:

$$
E_{o}=4 \pi R_{m}^{3} P_{0} / 3
$$

It yields a value of $\approx 1.1 \mathrm{~mJ} \ll 2.6 \mathrm{~mJ}$ of calculated value at the fibre tip. The difference can be explained by the difference in enthalpy of water in expanded vapor and some contribution due to heat conduction loss to the fibre tip and also some acoustic waves energy.

\subsection{Fibre-Film}

\section{1) Thermal coupling:}

Laser-metal surface interaction depends on the laser beam parameters; such as laser wavelength and pulse power density. In addition, the metal thermal and mechanical properties including microstructure, chemical composition, thermal conductivity and absorption are crucial factors. The interaction results are also affected by the surrounding medium. During the interaction process some energy is absorbed by resistive losses e.g. electron-phonon while the remainder is reflected. A longitudinal wave as an elastic wave with high irradiance can carry and redistribute radiation momentum through the film while it propagates. However, this to a larger extend depends on the dimensionless thermal coupling coefficient, $\eta_{t}$ which in the case of IR radiation is relatively low,

$$
\eta_{t}=\rho c l A T / E
$$

where density $\rho=2700 \mathrm{~kg} / \mathrm{m}^{-3}$, specific heat capacity $c=900 \mathrm{~J} \cdot \mathrm{kg}^{-1} \cdot \mathrm{c}^{-1}$, irradiated area $A=2 \times 10^{-7} \mathrm{~m}^{2}$, temperature at water boiling point $T=373$ and the input laser energy $E \approx 2.6 \mathrm{~mJ}$ (after interaction with the water volume element described above). Using these values in Equation (10) gives $\eta_{t} \approx 4.4 \times 10^{-3}(0.44 \%$ «1) i.e., only $1.2 \mathrm{~mJ}$ equivalent to $1.5 \times 10^{10} \mathrm{~W} \cdot \mathrm{m}^{-2}$ is now coupled to the surface of film. However, the intensity falls to $e^{-1}(37 \%)$ of its original value at skin depth calculated by [31]

$$
\delta_{s}=\left(\pi \sigma \mu_{r} \mu_{0} \nu\right)^{-1 / 2}=8 \mathrm{~nm}
$$


where $\sigma=1.5 \times 10^{10}(\Omega \cdot \mathrm{m})^{-1}$ is the electrical conductivity, $\mu_{r}, \mu_{0}$ are the relative and vacuum permeability and $v$ is the light frequency. The relation (11) does not apply to higher frequencies in the visible or higher. Now taking $\alpha_{A} \approx 50 \times 10^{6} \mathrm{~m}^{-1}$ (reflection at $2.75 \mu \mathrm{m} \approx 90 \%$ ), it gives $\delta_{0 A} \approx 20 \times 10^{-9} \mathrm{~m}$ and substituting it in relation (2), $\tau_{r} \approx 250$ ps and similarly from relation (3) we get $X_{T A} \approx 800 \mathrm{~nm} \ll \phi_{f} \approx$ $500 \mu \mathrm{m}$ (core diameter), where the subscript A stands for aluminium. Thus a 1-D model can be assumed and that one obtains a non-adiabatic where the generated heat is diffused within the metal film i.e., $\tau_{p} \gg \tau_{r}$ and $\delta_{0 A} \ll X_{T A}$. The consequences of this situation are: a) since, $\delta_{0 w} \approx 4 \mu \mathrm{m}<X_{w} \approx 5 \mu \mathrm{m}$, so no photons are reached the metal surface and only the heat and shock wave are expected to traverse the film, b) $X_{T A} \approx 800 \mathrm{~nm} \gg d_{A} \approx 80 \mathrm{~nm}$ (i.e. about $10 \%$ ), hence it is highly expected to have a uniform heat transfer through the film with minimum temperature difference. It is shown that such shock waves can travel up to $1676 \mathrm{~ms}^{-1}$ using a Nd:glass laser and a thin plastic coated metallic film [18]. As the bubble expands or contracts in water, mechanical work done, $W_{m}(\mathrm{~J})$ is transferred from bubble to the surrounding fluid and consequently, the kinetic energy transferred from the expanding vapor on the thin film surface. This in fact is the principle upon which the electromechanical microdevices such as micro actuators and pumps are based. Fraction of the $W_{m}$ is utilized to overcome the ambient pressure and also to raise the vapor/water interface surface area. Thus, the useful $W_{m}=\int_{0}^{t} P_{v} \frac{\mathrm{d} V(t)}{\mathrm{d} t} \mathrm{~d} t$ of the explosive vaporization is

$$
W_{m}=\int_{0}^{t}\left[P_{v}-P_{o}-\xi\left(\frac{32 \pi}{3 V(t)}\right)^{1 / 3}\right] \frac{\mathrm{d} V(t)}{\mathrm{d} t} \mathrm{~d} t
$$

where $\xi$ is water surface tension and by integrating the Equation (12) we obtain the corresponding power $(\mathrm{W}=\mathrm{J} / \mathrm{s})$

$$
W_{m} / \mathrm{d} t=\int_{0}^{t}\left[P_{v}-P_{o}-\xi\left(\frac{32 \pi}{3 V(t)}\right)^{1 / 3}\right] \frac{\mathrm{d} V(t)}{\mathrm{d} t} \mathrm{~d}
$$

\section{2) Surface temperature:}

The next step is to determine the temperature distribution which in turn produces stresses and strain elastic waves. Assuming that the incident power is sufficiently low such that it only raises the surface temperature without melting it and since in our case $\alpha_{A} \phi_{f} \gg 1$, therefore, the laser acts as a plane transient heat source. The absorbed energy in small element of area $A$ is

$$
\delta T(x)=\frac{1-R}{C \rho_{A} \delta_{s}} \int_{t} I(x, t) \mathrm{d} t
$$

where $R$ and $C$ are the aluminium specific heat capacity and radiation reflection from the surface. The temperature distribution across the surface is the same as the energy density distribution in the optical pulse. For 1-D treatment we have

$$
\frac{\partial^{2} T(z, t)}{\partial z^{2}}-\frac{1}{D_{t}} \frac{\partial T(z, t)}{\partial t}=-\frac{Q(z, t)}{K}
$$


where $Q$ is the heat produced per unit volume $\left(\mathrm{W} \cdot \mathrm{m}^{-3}\right)$. From Carslaw and Jaeger, the solution of Equation (15) is

$$
\begin{aligned}
& T(z, t)=\frac{2 I_{0}\left(D_{t} t\right)^{1 / 2}}{K} \operatorname{ierfc}\left(\frac{z}{2\left(D_{t} t\right)^{1 / 2}}\right) \\
& \operatorname{ierfc}(\psi)=\frac{1}{\sqrt{\pi}} \mathrm{e}^{-\psi^{2}}-\frac{2 \psi}{\sqrt{\pi}} \int_{\psi}^{\infty} \mathrm{e}^{-\psi^{2}} \mathrm{~d} \psi \\
& T(0, t)=\frac{2 I_{0}\left(D_{t} t\right)^{1 / 2}}{\sqrt{\pi} K}
\end{aligned}
$$

Substituting the values of aluminium, $I_{0}=1.5 \times 10^{10} \mathrm{~W} \cdot \mathrm{m}^{-2}, D_{t} \approx 4 \times 10^{-7}$ $\mathrm{m}^{2} \cdot \mathrm{s}^{-1} \cdot \mathrm{K} \approx 240 \mathrm{~W} \cdot \mathrm{m}^{-1} \cdot \mathrm{K}^{-1}$ in Equation (18) gives a value of $T \approx 301 \mathrm{~K}$ (i.e., $80 \%$ of boiling temperature $-373 \mathrm{~K}$ is transferred to the surface) $\ll$ melting temperature $T_{m} \approx 933 \mathrm{~K}$ of aluminium.

3) Heat transfer.

Schematic representation of heat transfer through the thin film is shown in Figure 7.

The amount of heat produced at the surface $(T 1)$ is transferred to the back of aluminium film at (T2) by parabolic Fourier heat conduction where the heating occurs instantaneously is given by

$$
Q_{A}=\frac{K A}{x} \Delta T
$$

For $Q_{A} \approx 1.2 \mathrm{~mJ}, \Delta T \approx 2 \times 10^{-6}{ }^{\circ} \mathrm{C}$. By definition this implies that $T 1 \approx T 2$ due to high conductivity and being thermally thin film so that there is virtually no heat loss. This is justified by the fact that Biot number (i.e., the ratio of the

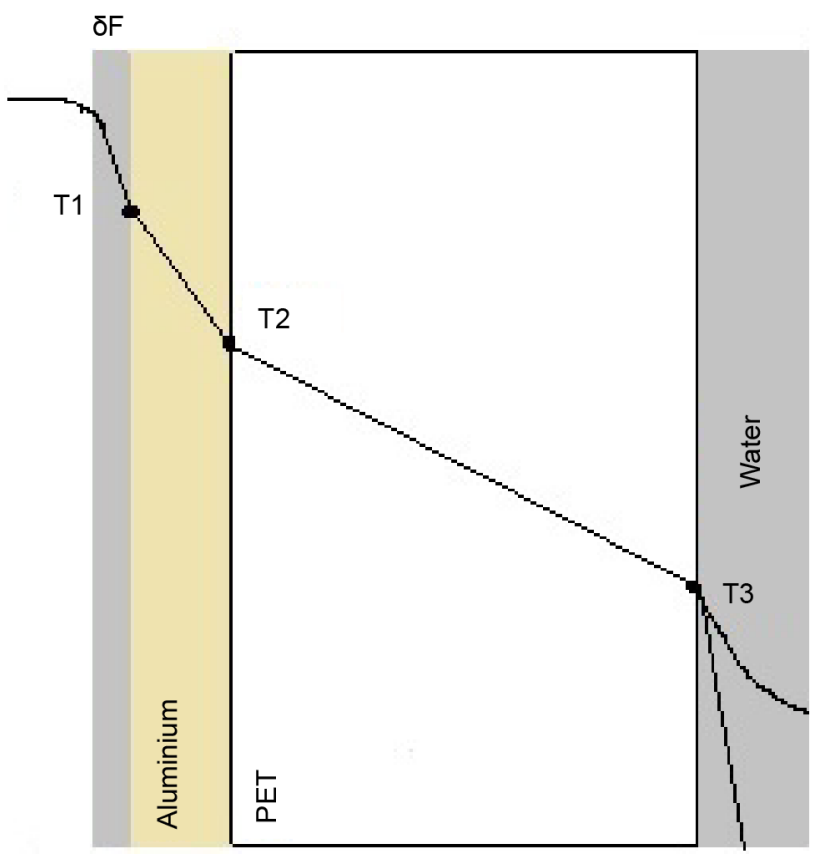

Figure 7. Schematic diagram of heat transfer through thin metal-PET film. 
resistance at the surface to within the film $)=h x / K \ll 0.1$ where $h\left(\mathrm{~W} \cdot \mathrm{m}^{2} \cdot \mathrm{K}^{-1}\right)$ is heat transfer coefficient for a single layer. The direct effect of thin metal coat on the polymer film is generation and faster transfer of heat and shock waves due to rapid thermal expansion and the explosive evaporation of the thin fluid layer and possibly the metallic layer. The amount of power transferred to back of polymer is approximately given by $\approx 8.8 \mathrm{~kW} \cdot \mathrm{e}^{-\alpha_{p} x_{p}} \approx 3 \mathrm{~kW}$ where $\alpha_{p} \approx 2 \times 10^{4}$ $\mathrm{m}^{-1}$ is the absorption coefficient of PET at $2.75 \mu \mathrm{m}$ and $x_{p}$ is the thickness. Figure 8 indicates the variation of heat transfer through the whole metal-polymer film represented by the ratio of vapor volumes. One implication of this result is that the maximum heat transfer occurs within $15 \mu$ s and then gradually decreases thereafter. Clearly, this varies with the condition of experiment in each case. It is noteworthy that $3 \mathrm{~kW}$ is very close to the last point on the curve at $200 \mu \mathrm{s}$ which is the final value of the thermal output.

However, for a multilayer film the overall heat transfer $(u)$ is defined as

$$
\frac{1}{u}=\frac{1}{h_{A}}+\frac{x_{A}}{K_{A}}+\frac{x_{p}}{K_{p}}+\frac{1}{h_{p}}
$$

where $h_{A}=K_{A} / \delta_{F}$ and $h_{p}=K_{p} / \delta_{F}$ and $\delta_{F}$ is the fluid thickness. Using the corresponding values one obtains athermal resistance per unit area of $1 / u \approx 3.3 \times$ $10^{-3} \mathrm{~m}^{2} \cdot \mathrm{K} \cdot \mathrm{W}^{-1}$. Thus $u \approx 3000 \mathrm{~W} \cdot \mathrm{m}^{-2} \cdot \mathrm{K}^{-1}$. Substituting this for Biot No. $\approx u \cdot \Sigma \mathrm{x} / \mathrm{K}$ $\approx 6.3 \times 10^{-3} \ll 0.1$, therefore, the overall film is also considered thermally thin. Finally, it is suggested that the work can be extended to nano or microscale possibly in conjunction with embedded nanoparticles within thin films or in the form of coated films where the mechanism of heat transfer is expected to be different to a bulk film.

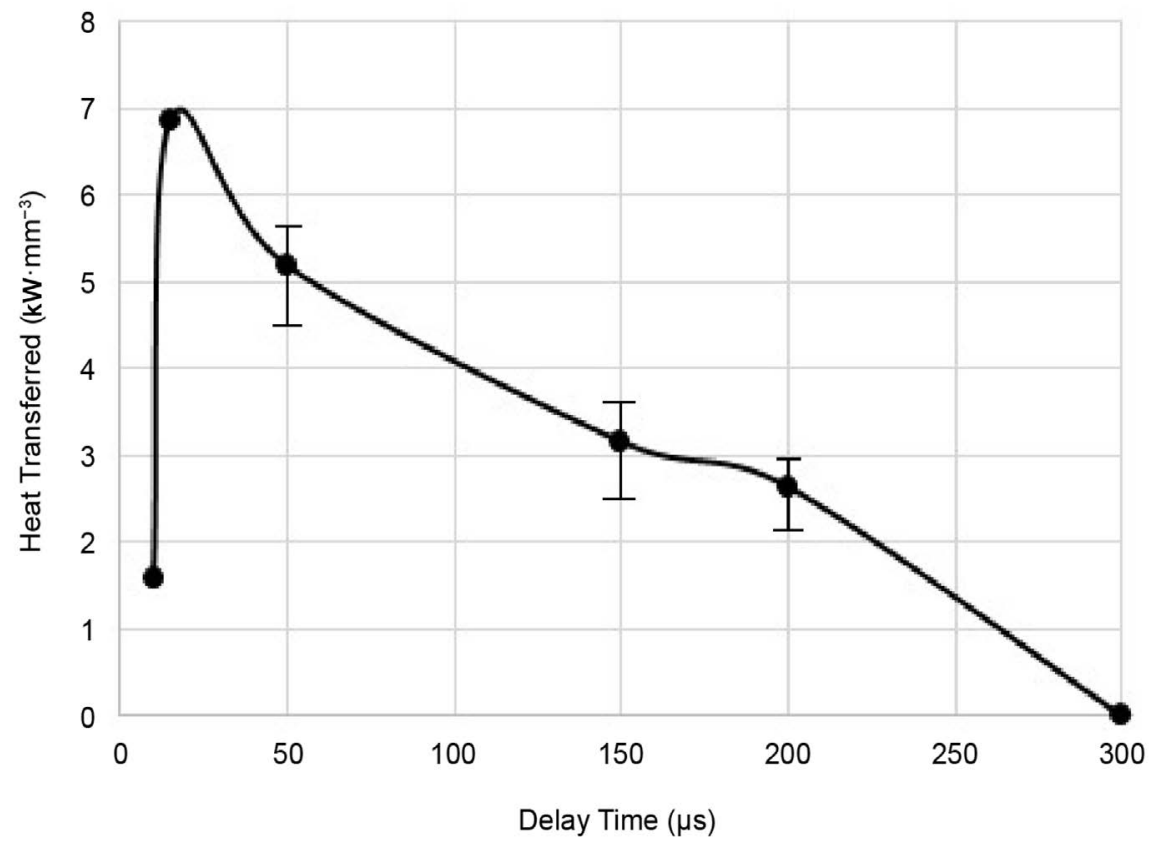

Figure 8. Calculated time-resolved heat transfer through the film. The maximum value occurs at $10 \mu$ s which then gradually decreases as the ratio of volumes is reduced. 


\section{Conclusion}

Mid-IR laser was used to study the heat transfer through metal-polymer film using shadowgraphy technique. The growth and collapse of cavitation bubble from fibre tip in contact with film demonstrated the heat transfer through the film with corresponding bubble volume. As distance increased to about $1 \mathrm{~mm}$, no heat transfer was observed. In our case, the Biot number in both the single aluminium layer and metal-polymer double film was less than 0.1 . Therefore, in both cases the overall film is considered as thermally thin. The thermal coupling of radiation was less than 1 and that temperature at the surface of metal was far below its melting temperature. The practical application of such situation plays a key role in number of scientific, engineering and biomedical applications.

\section{References}

[1] Marcus, S and Lowder, J. (1975) Impulsive Loading of Targets by HF Laser Pulses. Journal of Applied Physics, 46, 2293-2294. https://doi.org/10.1063/1.321824

[2] Deka, B., Dyer, P.E. and Sayers, J. (1980) Investigation of Laser Supported Detonation Waves and Thermal Coupling Using $2.8 \mu \mathrm{m}$ HF Laser Irradiation Metal Targets. Journal de Physique, 11, C9-75-C98.

[3] Dewhurst, R., Nurse, A. and Palmer, S. (1998) High Power Optical Fibre Delivery System for the Laser Generation of Ultrasound. Ultrasonics, 26, 307-310. https://doi.org/10.1016/0041-624X(88)90027-3

[4] Bulgakova, N. and Bulgakov, A. (2001) Pulsed Laser Ablation of Solids: Transition from Normal Vaporization to Phase Explosion. Applied Physics A, 73, 199-208. https://doi.org/10.1007/s003390000686

[5] Khosroshahi, M.E., Mahmoodi, M. and Tavakoli, J. (2007) Characterization of Ti6Al4V Implant Surface Treated by Nd:YAG Laser and Emery Paper for Orthopaedic Applications. Applied Surface Science, 253, 8772-8781. https://doi.org/10.1016/j.apsusc.2007.04.084

[6] Khosroshahi, M.E., Anoosheh Pour, F., Hadavi, M. and Mahmoodi, M. (2010) In Situ Monitoring the Pulse $\mathrm{CO}_{2}$ Laser Interaction with 316-L Stainless Steel Using Acoustical Signals and Plasma Analysis. Applied Surface Science, 256, 7421-7427. https://doi.org/10.1016/j.apsusc.2010.05.083

[7] Ward, B. and Emmony, D. (1991) Direct Observation of the Pressure Developed in a Liquid During Cavitation Bubble Collapse. Applied Physics Letters, 59, 2228-2230. https://doi.org/10.1063/1.106078

[8] Dyer, P.E., Khosroshahi, M.E. and Tuft, S. (1993) Studies of Laser-Induced Cavitation and Tissue Ablation in Saline Using a Fibre-Delivered Pulsed HF Laser. Applied Physics B, 56, 84-93. https://doi.org/10.1007/BF00325245

[9] Ith, M., Pratisto, H., Altermatt, H. Frenz. M. and Webr, H. (1994) Dynamics of Laser-Induced Channel Formation in Water and Influence of Pulsed Duration on the Ablation of Tissue Underwater with Pulsed Erbium Laser Radiation. Applied Physics B, 59, 621-629. https://doi.org/10.1007/BF01081183

[10] Xianzeng, Z., Chuanguo, Ch., Faner, Ch. and Zhenlin, Z. (2016) In Vitro Investigation on Ho:YAG Laser-Assisted Bone Ablation Underwater. Lasers in Medical Science, 31, 891-898. https://doi.org/10.1007/s10103-016-1931-x

[11] Hetiz, J., Arenhoilz, E., Kefer, T., Bauerle, D., et al. (1992) Enhanced Adhesion of 
Metal Films on PET after UV-Laser Treatment. Applied Physics A, 55, 391-392. https://doi.org/10.1007/BF00324090

[12] Zewig, A. and Deutsch, T. (1992) Shock Waves Generated by Confined XeCl Excimer Laser Ablation of Polyimide. Applied Physics B, 54, 76-82.

https://doi.org/10.1007/BF00331737

[13] Kreuusing, A. (2004) Underwater and Water-Assisted Laser Processing: Part 2-Etching, Cutting and Rarely Used Methods. Optics and Lasers in Engineering, 41, 329-352. https://doi.org/10.1016/S0143-8166(02)00143-4

[14] Toker, G., Bulatov, V., Kovalchuk, T. and Schechter, I. (2009) Underwater Interaction of $1064 \mathrm{~nm}$ Laser Radiation with Metal Target. World Academy of Science, Engineering and Technology, 55, 25-28.

[15] Nath, A., Hansden, D., Roy, S. and Choudery, A. (2010) A Study on Laser Drilling of Thin Film Sheet in Air and Underwater. Journal of Applied Physics, 107, Article ID: 123103.

[16] Wang, J., Weaver, R. and Sottos, N. (2002) A Parametric Study of Laser Induced Thin Film Spallation. Experimental Mechanics, 42, 74-83.

https://doi.org/10.1007/BF02411054

[17] Bostanjoglo, O. and Nick, T. (1996) Hydrodynamic Instabilities in Laser-Produced Melts of Metal Films. Journal of Applied Physics, 79, 8726-8729. https://doi.org/10.1063/1.362499

[18] Harith, M., Palleschi, V., Salvetti, A., et al. (1989) Dynamics of Laser-Induced Driven Shock Waves in Water. Applied Physics Letters, 66, 5194-5197.

[19] Dyer, P.E., Karnakis, D. and Sands, D. (1997) Fast Photography of UV Laser Ablated Metal Films. Applied Surface Science, 109, 168-173. https://doi.org/10.1016/S0169-4332(96)00654-X

[20] Siew, W., Tou, T. and Wong, K. (2005) Shadowgraphy of Pulsed Co2 Laser Ablation of Polymers. Applied Surface Science, 248, 281-285. https://doi.org/10.1016/j.apsusc.2005.03.052

[21] Ambrosin, D. and Rastogi, P. (2006) Optical Methods in Heat Transfer and Fluid Flow. Optics and Lasers in Engineering, 44, 155-158. https://doi.org/10.1016/j.optlaseng.2005.07.005

[22] Astarita, T., Cardone and Carlomagno, G. (2006) IR Thermography: An Optical Method in Heat Transfer and Fluid Flow Visualization. Optics and Lasers in Engineering, 44, 261-281. https://doi.org/10.1016/j.optlaseng.2005.04.006

[23] Fardel, R., Nagel, M., Nuesch, F., et al. (2009) Shadowgraphy Investigation of Laser-Induced Forward Transfer: Front Side and Back Side Ablation of the Triazene Polymer Sacrificial Layer. Applied Surface Science, 255, 5430-5434. https://doi.org/10.1016/j.apsusc.2008.07.187

[24] Kononenko, T., Alloncle, P., Konov, V. and Sentis, M. (2011) Shadowgraphic Imaging of Laser Transfer Driven by Metal Film Blistering. Applied Physics A, 102, 49-54. https://doi.org/10.1007/s00339-010-6083-4

[25] Tanda, G., Fossa, M. and Misale, M. (2014) Heat Transfer in Water Using a Schliern Technique. International Journal of Heat and Mass Transfer, 71, 451-458. https://doi.org/10.1016/j.ijheatmasstransfer.2013.12.022

[26] Hihath, S., Santala, M., Cen, X., et al. (2015) High Speed Direct Imaging of Thin Metal Film Ablation by Movie-Mode Dynamic Transmission Electrom Microscopy. Scientific Reports, 6, 1-5.

[27] Khosroshahi, M.E. and Ghasemi, A. (2004) Interaction Studies of Multimode Pulsed 
HF Laser with Enamel Tissue using Photothermal Deflection and Spectroscopy. Lasers in Medical Science, 18, 196-203.

[28] Hale, G. and Query, M. (1973) Optical Constants of Water in the $200 \mathrm{~nm} 200 \mu \mathrm{m}$ Wavelengths Region. Applied Optics, 12, 555-563.

https://doi.org/10.1364/AO.12.000555

[29] Cross, F., Al-Dhair, R. and Dyer, P.E. (1988) Ablative and Acoustic Response of Pulsed UV Laser-Irradiated Vascular Tissue in a Liquid Environment. Journal of Applied Physics, 64, 2194-2197. https://doi.org/10.1063/1.341707

[30] Young, F.R. (1989) Cavitation. McGraw-Hill, Oxford.

[31] Bleaney, B.I. and Bleaney, B. (1965) Electricity and Magnetism. 2nd Edition, Oxford Univ. Press, Oxford. 